\title{
PROPRIEDADES TERMOFÍSICAS DA POLPA DO CUPUAÇU COM DIFERENTES TEORES DE SÓLIDOS ${ }^{1}$
}

\author{
Termophysical properties of cupuaçu pulp at different solids contents
}

\author{
Josalice de Lima Araújo², Alexandre José de Melo Queiroz ${ }^{3}$, Rossana Maria Feitosa de Figueirêdo ${ }^{3}$
}

\section{RESUMO}

Este trabalho foi realizado com o objetivo de determinar as propriedades termofísicas difusividade térmica, calor específico e condutividade térmica da polpa do cupuaçu em três condições de teor de sólidos: integral $\left(12^{\circ} \mathrm{Brix}\right)$, $9^{\circ} \mathrm{Brix}$ e com eliminação parcial dos sólidos insolúveis (peneirado). A difusividade térmica foi determinada por meio do aparato de Dickerson (1965); para o calor específico, utilizou-se a metodologia do calorímetro de mistura, e a condutividade térmica foi determinada considerando-se a relação entre a difusividade térmica, o calor específico e a massa específica. Foram utilizadas equações para o cálculo teórico dessas propriedades e os resultados foram comparados com os valores experimentais. Os resultados experimentais médios, obtidos para as difusividades térmicas, calores específicos e condutividades térmicas das amostras integral, $9^{\circ}$ Brix e peneiradas foram, respectivamente: $1,31 \times 10^{-7}, 1,32 \times 10^{-7}$ e $1,27 \times 10^{-7} \mathrm{~m}^{2} / \mathrm{s} ; 3,24,3,71$ e $3,18 \mathrm{~kJ} / \mathrm{kg}^{\circ} \mathrm{C}$; e $0,44 \mathrm{~W} / \mathrm{m}^{\circ} \mathrm{C}$, $0,50 \mathrm{~W} / \mathrm{m}^{\circ} \mathrm{C}$ e $0,42 \mathrm{~W} / \mathrm{m}^{\circ} \mathrm{C}$.

Termos para indexação: Termofísica, fruta, Theobroma grandiflorum.

\begin{abstract}
This work was accomplished with the aim of determining the termophysical properties, thermal diffusivity, specific heat and thermal conductivity of cupuaçu pulp in three conditions of solids contents: $12^{\circ} \mathrm{Brix}, 9^{\circ} \mathrm{Brix}$, and partial elimination of the insoluble solids (sieving). The thermal diffusivity was determined by means of Dickerson's (1965) apparatus, for the specific heat the method of mixtures was used and the thermal conductivity was determined starting from the relationship among thermal diffusivity, specific heat and density. Equations were used for the theoretical calculation of those properties and the results were compared with the experimental values. The average results, obtained for the thermal diffusivity, specific heat and thermal conductivity of the $12^{\circ} \mathrm{Brix}, 9^{\circ} \mathrm{Brix}$ and sieved samples were, respectively: $1.31 \times 10^{-7}, 1.32 \times 10^{-7}$ and $1.27 \times 10^{-7} \mathrm{~m}^{2} / \mathrm{s}$; $3.24,3.71$ and $3.18 \mathrm{~kJ} / \mathrm{kg}^{\circ} \mathrm{C}$; and $0.44 \mathrm{~W} / \mathrm{m}^{\circ} \mathrm{C}, 0.50 \mathrm{~W} / \mathrm{m}^{\circ} \mathrm{C}$ and $0.42 \mathrm{~W} / \mathrm{m}^{\circ} \mathrm{C}$.
\end{abstract}

Index terms: Thermophysical, fruit, Theobroma grandiflorum.

(Recebido para publicação em 10 de setembro de 2002 e aprovado em 10 de junho de 2003)

\section{INTRODUÇÃO}

Polpas de frutas, na forma de matéria-prima destinada à industrialização, estão, como outros produtos alimentícios, sujeitas a processos de aquecimento e resfriamento. Os tratamentos térmicos necessários para eliminar microrganismos contaminantes, inativar enzimas naturais e retardar processos metabólicos e microbiológicos em condições de estocagem têm utilidade ainda nos projetos de equipamentos aquecedores, resfriadores, evaporadores, separadores e embaladores dos produtos. Em todos esses casos são necessários conhecimentos das propriedades termofísicas das matérias-primas, a fim de se calcular cargas térmicas, dispêndios energéticos, dimensionamento dos equipamentos, etc.
$\mathrm{O}$ uso das equações de transferência de calor e massa é condicionado à disponibilidade de dados sobre as características intrínsecas dos materiais envolvidos, em particular das propriedades termofísicas. Estimativas de dispêndios e trocas energéticas podem ser realizadas a partir dos valores de massa específica, difusividade térmica, calor específico e condutividade térmica dos materiais.

A difusividade térmica é definida como a relação entre a condutividade térmica e o calor específico do produto multiplicado por sua massa específica; em termos físicos, dá uma medida de como a temperatura pode variar quando um material é submetido a uma situação de aquecimento ou resfriamento (LEWIS, 1993). A difusividade térmica, descrita ainda como a razão entre a habilidade em transferir calor e a capacidade de armazenar calor,

1. Parte da dissertação do primeiro autor apresentada àUniversidade Federal da Paraíba.

2. Químico Industrial, Av. Aprígio Veloso, 759, Bodocongó, Caixa Postal 10017-58109-970 - Campina Grande, Paraíba.

3. Engenheiro Agrícola - UFPB, Caixa Postal 10017 - 58109-970 - Campina Grande, PB. 
em situações em que a transferência de calor ocorre em regime transiente, tem lugar como a mais utilizada das propriedades térmicas.

O calor específico, cujo valor é significativamente afetado pela quantidade de água presente no material e pelo estado físico desta água (LEWIS, 1993), é entendido como a quantidade de calor necessária para alterar a temperatura de uma unidade de massa, sendo, portanto, fundamental para se determinar a quantidade de energia a ser adicionada ou removida nos processos de aquecimento e resfriamento.

A condutividade térmica depende principalmente da composição do produto, incluindo a presença de espaços vazios e do grau de homogeneidade estrutural. A condutividade térmica de um material, de acordo com Rao e Rizvi (1994), é a medida da sua capacidade para conduzir calor. No caso de condução unidimensional de calor, a equação proposta por Fourier (ÖZISIK, 1977) e conhecida como a equação fundamental de transferência de calor exprime a relação da condutividade térmica com a transferência de calor e a temperatura.

O cupuaçu (Theobroma grandiflorum Schum.), uma das frutas mais populares da Amazônia, é consumido sob diferentes formas, seja como doces, geléias, licores, compotas, sorvetes (CALZAVARA et al., 1984), seja como polpa ou sucos com os mais diversos teores de sólidos. Nessas apresentações constitui-se em matéria-prima potencial para a indústria, o que conduz à necessidade de se conhecer as características e propriedades desses materiais. Neste trabalho, objetivou-se a determinação da difusividade térmica, do calor específico e da condutividade térmica de amostras de polpa de cupuaçu com três teores de sólidos: polpa integral $\left(12^{\circ}\right.$ Brix $)$, polpa a $9^{\circ}$ Brix e polpa com teor reduzido de sólidos insolúveis (polpa peneirada).

\section{MATERIAL E MÉTODOS}

A polpa de cupuaçu integral $\left(12^{\circ} \mathrm{Brix}\right)$ foi obtida pelo processamento de frutos maduros. Por meio da diluição dessa polpa com água destilada, foram elaboradas as amostras denominadas " $9{ }^{\circ}$ Brix". As polpas "peneiradas" (12 ${ }^{\circ}$ Brix) foram obtidas fazendo-se passar as amostras integrais em peneiras com malhas de $0,59 \mathrm{~mm}$.

A difusividade térmica das diferentes amostras de cupuaçu foi determinada com o auxílio de um equipamento semelhante ao utilizado por Dickerson (1965), cuja montagem consiste em uma cápsula metálica cilíndrica com termopares conectados a um indicador de temperatura e um banho termostático com agitação (Figura 1). As dimensões do cilindro eram de 0,049 $\mathrm{m}$ de diâmetro interno, 0,30 $\mathrm{m}$ de comprimento externo e $0,23 \mathrm{~m}$ de comprimento útil interno. Em cada ensaio, a cápsula era preenchida com as amostras de polpa de cupuaçu e mergulhada no banho termostático com agitação mecânica. No instante em que o banho era ligado (temperatura ambiente, em torno de $25^{\circ} \mathrm{C}$ ), iniciavam-se as leituras das temperaturas no centro $\left(\mathrm{T}_{\mathrm{c}}\right)$ e na superfície do cilindro $\left(\mathrm{T}_{\mathrm{s}}\right)$, em intervalos de 2 minutos, até o final do processo, após 60 minutos, a uma temperatura de cerca de $60^{\circ} \mathrm{C}$. Os valores da taxa constante de aquecimento (A) foram obtidos pelo coeficiente angular da reta da regressão linear do gráfico de temperatura versus tempo, construído com esses dados experimentais. $\mathrm{O}$ cálculo da difusividade térmica, na faixa de temperatura entre $25^{\circ} \mathrm{C}$ e $60^{\circ} \mathrm{C}$, pode ser determinado utilizando-se a Equação (1) proposta por Dickerson (1965):

$$
\dot{a}=\frac{\mathrm{AR}_{\mathrm{c}}{ }^{2}}{4\left(\mathrm{~T}_{\mathrm{s}}-\mathrm{T}_{\mathrm{c}}\right)}
$$

Em que: $\alpha=$ difusividade térmica $\left(\mathrm{m}^{2} / \mathrm{s}\right), \mathrm{A}=$ taxa constante de aquecimento $\left({ }^{\circ} \mathrm{C} / \mathrm{s}\right), \mathrm{R}_{\mathrm{c}}=$ raio do cilindro $(\mathrm{m}), \mathrm{T}_{\mathrm{s}}=$ temperatura na superfície do cilindro de raio $\mathrm{R}\left({ }^{\circ} \mathrm{C}\right), \mathrm{T}_{\mathrm{c}}=$ temperatura no centro do cilindro $\left({ }^{\circ} \mathrm{C}\right)$.

Além das determinações experimentais, foram determinadas as difusividades térmicas utilizando-se as equações desenvolvidas por Riedel (1969), Martens, citado por Singh (1982) e Choi e Okos (1983), apresentadas na Tabela 1.

O calor específico foi determinado com a utilização de um calorímetro de mistura, construído com uma ampola de garrafa térmica comercial, com capacidade para 1000 $\mathrm{mL}$, envolvida por uma camada de $0,05 \mathrm{~m}$ de isolante térmico constituído de lã de vidro e tendo como invólucro um cilindro de PVC de 0,15 m de diâmetro (Figura 2). O fechamento da ampola foi feito com uma rolha de borracha, por meio da qual foi introduzida a haste de um termopar com precisão de $0,1^{\circ} \mathrm{C}$. Para as determinações do calor específico, os materiais foram embalados em sacos de polietileno de baixa densidade, termosselados e imersos em um banho termostático a uma temperatura de $30^{\circ} \mathrm{C}$, por um período de aproximadamente $30 \mathrm{~min}$. O calor específico foi calculado com o uso da Equação 2: 


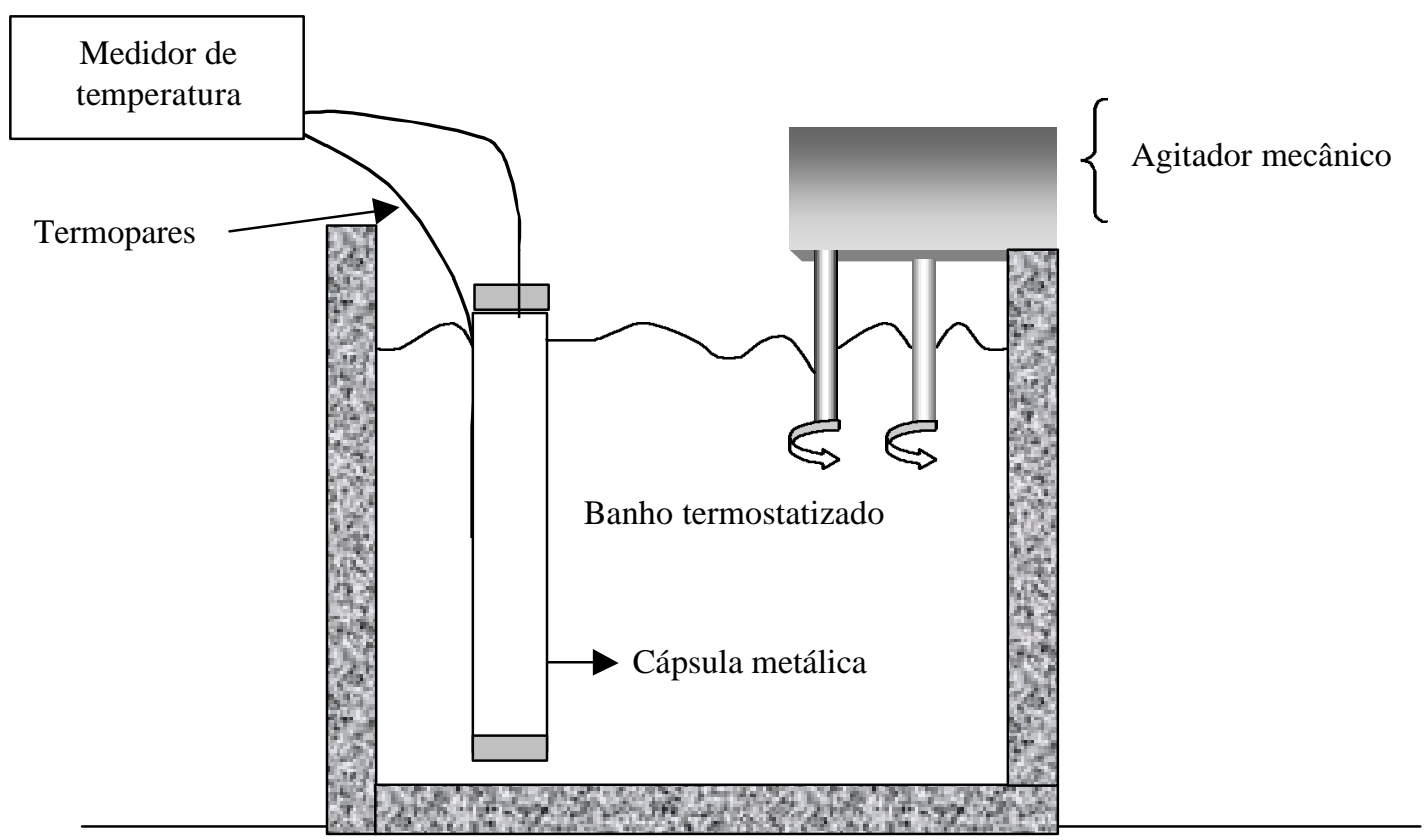

FIGURA 1 - Esquema do aparato utilizado para a determinação da difusividade térmica

TABELA 1 - Equações para o cálculo de difusividade térmica de produtos alimentícios.

\begin{tabular}{ll}
\hline \multicolumn{1}{c}{ Produto } & \multicolumn{1}{c}{ Equação } \\
\hline Alimentos em geral (RIEDEL, 1969) & $\alpha=0,088 \cdot 10^{-6}+\left(\alpha_{\mathrm{w}}-0,088 \cdot 10^{-6}\right) \mathrm{X}_{\mathrm{w}}$ \\
\hline Alimentos em geral (MARTENS, citado por SINGH, 1982) & $\alpha=\left[0,057363 \mathrm{X}_{\mathrm{w}}+0,000288(\mathrm{~T}+273)\right] \cdot 10^{-6}$ \\
\hline & $\alpha=\alpha_{\mathrm{w}} \mathrm{X}_{\mathrm{w}}+\alpha_{\mathrm{s}} \mathrm{X}_{\mathrm{s}}$ \\
Suco de tomate (CHOI e OKOS, 1983) & $\alpha_{\mathrm{w}}=\left[0,1363+0,0004875 \mathrm{~T}-0,000001632 \mathrm{~T}^{2}\right] .10^{-6}$ \\
& $\alpha_{\mathrm{s}}=\left[0,009508+0,000234 \mathrm{~T}+0,000001761 \mathrm{~T}^{2}\right] 10^{-6}$ \\
\hline
\end{tabular}

Em que: $\alpha$ = difusividade térmica $\left(\mathrm{m}^{2} / \mathrm{s}\right), \alpha_{\mathrm{w}}=$ difusividade térmica da água $\left(\mathrm{m}^{2} / \mathrm{s}\right), \alpha_{\mathrm{s}}=$ difusividade térmica dos sólidos $\left(\mathrm{m}^{2} / \mathrm{s}\right), \mathrm{X}_{\mathrm{w}}=$ fração mássica da água (adimensional), $\mathrm{X}_{\mathrm{s}}=$ fração mássica dos sólidos (adimensional), $\mathbf{T}=$ temperatura $\left({ }^{\circ} \mathrm{C}\right)$.

$$
\mathrm{Cp}_{\mathrm{a}}=\frac{\mathrm{Cp}_{\mathrm{w}} \cdot \mathrm{M}_{\mathrm{w}} \cdot\left(\mathrm{T}_{\mathrm{e}}-\mathrm{T}_{\mathrm{w}}\right)+\mathrm{C}_{\mathrm{cal}} \cdot\left(\mathrm{T}_{\mathrm{e}}-\mathrm{T}_{\mathrm{i}}\right)}{\mathrm{M}_{\mathrm{a}} \cdot\left(\mathrm{T}_{\mathrm{i}}-\mathrm{T}_{\mathrm{e}}\right)}
$$

Em que: $\mathrm{Cp}_{\mathrm{a}}=$ calor específico da amostra $\left(\mathrm{kJ} / \mathrm{kg}^{\circ} \mathrm{C}\right)$, $\mathrm{Cp}_{\mathrm{w}}=$ calor específico da água $\left(\mathrm{kJ} / \mathrm{kg}^{\circ} \mathrm{C}\right), \mathrm{M}_{\mathrm{w}}=$ massa de água adicionada $(\mathrm{kg}), \mathrm{T}_{\mathrm{e}}=$ temperatura de equilíbrio $\left({ }^{\circ} \mathrm{C}\right), \mathrm{T}_{\mathrm{w}}=$ temperatura inicial da água $\left({ }^{\circ} \mathrm{C}\right), \mathrm{C}_{\mathrm{cal}}=$ capacidade calorífica do calorímetro $\left(\mathrm{kJ} /{ }^{\circ} \mathrm{C}\right), \mathrm{M}_{\mathrm{c}}=$ massa do calorímetro $(\mathrm{kg}), \mathrm{T}_{\mathrm{i}}=$ temperatura inicial da amostra e do calorímetro $\left({ }^{\circ} \mathrm{C}\right), \mathrm{M}_{\mathrm{a}}=$ massa da amostra $(\mathrm{kg})$.
Por meio de equações desenvolvidas por outros autores, calcularam-se também os calores específicos das amostras utilizando-se as equações (Tabela 2) propostas por Dickerson (1968), Siebel, citado por Choi e Okos (1986) e Lamb, citado por Choi e Okos (1986).

As condutividades térmicas (k) das amostras foram calculadas pela relação entre a difusividade térmica $(\alpha)$, o calor específico $\left(C_{p}\right)$ e a massa específica $(\rho)$, expressa pela Equação (3). 


$$
\alpha=\frac{\mathrm{k}}{\rho \mathrm{c}_{\mathrm{p}}}
$$

Os valores de massa específica das amostras de polpa de cupuaçu integral, $9^{\circ}$ Brix e peneirada na temperatura de $30^{\circ} \mathrm{C}$ utilizados no cálculo da condutividade térmica foram os obtidos por Araújo (2001) utilizando picnômetro de $25 \mathrm{~mL}$, e são, respectivamente, $1.027,6 \pm 0,29 \mathrm{~kg} / \mathrm{m}^{3}, 1.017,1 \pm 0,72 \mathrm{~kg} / \mathrm{m}^{3} \mathrm{e}$ $1.039,0 \pm 0,55 \mathrm{~kg} / \mathrm{m}^{3}$.
Também foram calculadas condutividades térmicas utilizando-se correlações apresentadas na literatura por Sweat e Parmelee (1978), Riedel, citado por Choi e Okos (1986) e Choi e Okos (1983), apresentadas na Tabela 3.

As análises estatísticas dos dados de difusividade térmica, calor específico e condutividade térmica foram feitas por delineamento inteiramente casualizado, com a utilização do software ASSISTAT, versão 6.2 (SILVA, 1996).

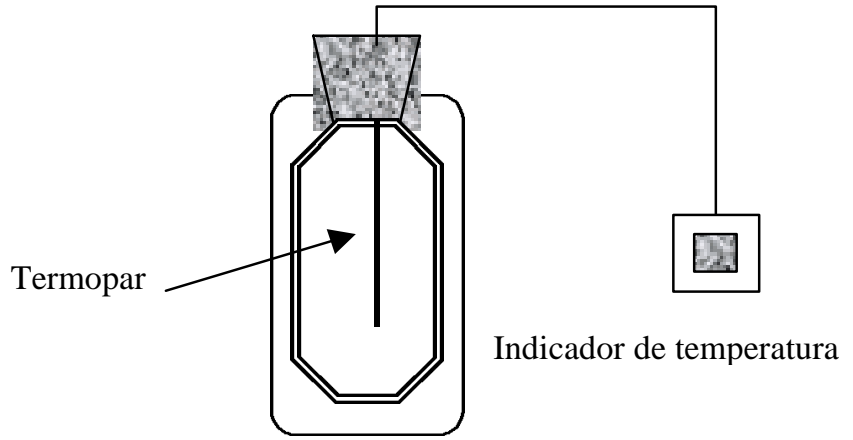

FIGURA 2 - Desenho do calorímetro de mistura

TABELA 2 - Equações para o cálculo do calor específico de produtos alimentícios.

\begin{tabular}{ccc}
\hline Produto & Autor & Equação \\
\hline Produtos alimentícios & Siebel & $\mathrm{C}_{\mathrm{p}}=0,837+3,349 \mathrm{X}_{\mathrm{w}}$ \\
Produtos alimentícios & Dickerson & $\mathrm{C}_{\mathrm{p}}=1,675+2,512 \mathrm{X}_{\mathrm{w}}$ \\
Gêneros alimentícios & Lamb & $\mathrm{C}_{\mathrm{p}}=1,465+2,721 \mathrm{X}_{\mathrm{w}}$ \\
\hline
\end{tabular}

Em que: $C_{p}=$ calor específico $\left(\mathrm{kJ} / \mathrm{kg}^{\mathrm{0}} \mathrm{C}\right), X_{\mathrm{w}}=$ fração mássica da água (adimensional)

TABELA 3 - Equações para o cálculo de condutividade térmica de produtos alimentícios.

\begin{tabular}{ll}
\hline \multicolumn{1}{c}{ Produto } & \multicolumn{1}{c}{ Equação } \\
\hline $\begin{array}{l}\text { Sucos de frutas, leite e solução de açúcar (RIEDEL, } \\
\text { citado por CHOI e OKOS, 1986) }\end{array}$ & $\mathrm{k}=\left[326,58+1,0412 \mathrm{~T}-0,00337 \mathrm{~T}^{2}\right] \cdot\left[0,46+0,54 \mathrm{X}_{\mathrm{w}}\right] 1,73 \cdot 10^{-3}$ \\
\hline & $\mathrm{k}=\mathrm{k}_{\mathrm{w}} \mathrm{X}_{\mathrm{w}}+\mathrm{k}_{\mathrm{s}} \mathrm{X}_{\mathrm{s}}$ \\
Suco de tomate (CHOI e OKOS, 1983) & $\mathrm{k}_{\mathrm{w}}=0,5747+0,001723 \mathrm{~T}-0,000006722 \mathrm{~T}^{2}$ \\
& $\mathrm{k}_{\mathrm{s}}=0,5998+0,001593 \mathrm{~T}+0,00000163 \mathrm{~T}^{2}$ \\
\hline $\begin{array}{l}\text { Margarina e derivados de leite (SWEAT e } \\
\text { PARMELEE, 1978) }\end{array}$ & $\mathrm{k}=0,141+0,412 \mathrm{X}_{\mathrm{w}}$
\end{tabular}

Em que: $k=$ condutividade térmica $\left(W / m^{\circ} \mathrm{C}\right), k_{w}=$ condutividade térmica da água $\left(W / m^{\circ} C\right), k_{s}=$ condutividade térmica dos sólidos $\left(\mathrm{W} / \mathrm{m}^{\circ} \mathrm{C}\right), \mathrm{X}_{\mathrm{w}}=$ fração mássica da água (adimensional), $\mathrm{X}_{\mathrm{s}}=$ fração mássica dos sólidos (adimensional), $\mathrm{T}=$ temperatura $\left({ }^{\circ} \mathrm{C}\right)$ 
O critério utilizado para avaliar o ajuste de cada equação aos dados experimentais foi o erro percentual, apresentado na Equação 4.

$$
\varepsilon_{p}=\left|\frac{\mathrm{V}_{\mathrm{e}}-\mathrm{V}_{\mathrm{p}}}{\mathrm{V}_{\mathrm{e}}}\right| \times 100
$$

Em que: $\varepsilon_{\mathrm{p}}=$ erro percentual, $\mathrm{V}_{\mathrm{e}}=$ valor experimental, $\mathrm{V}_{\mathrm{p}}=$ valor predito.

\section{RESULTADOS E DISCUSSÃO}

Na Tabela 4, são mostrados os valores médios de difusividade térmica das polpas de cupuaçu, calculados pelos parâmetros $\mathrm{A}, \mathrm{T}_{\mathrm{s}}-\mathrm{T}_{\mathrm{c}}$ e $\mathrm{R}_{\mathrm{c}}$. A comparação entre as médias das amostras integral, $9^{\circ}$ Brix e peneirada, utilizando o teste de Tukey, não demonstrou diferença significativa entre as amostras a 5\% de probabilidade. Isso significa que as diferenças entre os teores de sólidos das amostras não resultaram em influência sobre a difusividade térmica.

Os valores de difusividade térmica determinados experimentalmente de $1,27 \times 10^{-7}$ a $1,32 \times 10^{-7}$ $\mathrm{m}^{2} / \mathrm{s}$ encontram-se dentro da faixa de valores obtidos por autores como Alvarado (1994), que trabalhou com polpa de mamão e Bhowmik e Hayakawa (1979), com polpa de maçã e tomate, os quais obtiveram resultados de difusividade térmica variando de $1 \times 10^{-7} \mathrm{~m}^{2} / \mathrm{s}$ a $2 \times 10^{-7} \mathrm{~m}^{2} / \mathrm{s}$.

Os resultados para polpa de cupuaçu também se encontram na mesma faixa de valores determinados para polpas de manga, estudadas por Simões (1997). De acordo com esse autor, também não foram verificadas diferenças significativas nas difusi- vidades térmicas das polpas de manga integral, centrifugada e peneirada.

Na Tabela 5, são apresentados os valores de difusividade térmica obtidos a partir de equações da literatura, calculados considerando-se a difusividade térmica da água igual a $1,48 \times 10^{-7} \mathrm{~m}^{2} / \mathrm{s}$, temperatura de $30^{\circ} \mathrm{C}$ e umidades de $84,92 \%, 88,05 \%$ e $84,79 \%$ para as amostras integral, $9^{\circ}$ Brix e peneirada, respectivamente. Comparando os valores da difusividade térmica obtidos pelas equações de Riedel (1969), Choi e Okos (1983) e Martens, citado por Singh (1982) com os dados experimentais, verifica-se que os resultados preditos pelas equações apresentaram valores superiores, exceto para a difusividade térmica da polpa integral calculada pela equação de Choi e Okos (1983).

Observando os erros em todas as amostras, determinados para as três equações, constata-se que seus valores variaram numa faixa entre $0,76 \%$, para o menor erro, e $8,66 \%$, para o maior. O erro para o material integral, resultante da utilização da equação de Choi e Okos (1983), foi menor do que com as demais equações. Também para o material $9^{\circ}$ Brix, os erros resultantes da utilização da equação de Choi e Okos (1983) foram menores que os verificados nas demais equações, chegando a 1/4 do erro obtido com a utilização da equação de Riedel (1969). Os erros determinados para a polpa peneirada, com as equações de Riedel (1969) e Martens, citado por Choi e Okos (1986), resultaram superiores a $5 \%$, ao passo que para a equação de Choi e Okos (1983), o erro verificado foi inferior em mais de quatro vezes em relação aos erros determinados com as demais equações. Do conjunto de resultados, o que se observam são boas aproximações obtidas a partir dos valores preditos, empregando-se as correlações citadas.

TABELA 4 - Difusividade térmica da polpa de cupuaçu.

\begin{tabular}{cccc}
\hline Amostra & $\mathbf{A}\left({ }^{\mathbf{0}} \mathbf{C} / \mathbf{m i n}\right)$ & $\mathbf{T}_{\mathbf{s}}-\mathbf{T}_{\mathbf{c}}\left({ }^{\mathbf{0}} \mathbf{C}\right)$ & Difusividade Térmica $\left(\mathbf{m}^{\mathbf{2}} / \mathbf{s}\right)$ \\
\hline Polpa integral & 0,52 & 10,0 & $1,31 \times 10^{-7} \pm 0,06 \times 10^{-7} \mathrm{a}$ \\
Polpa a $9^{\circ}$ Brix & 0,52 & 9,8 & $1,32 \times 10^{-7} \pm 0,04 \times 10^{-7} \mathrm{a}$ \\
Polpa peneirada & 0,50 & 9,8 & $1,27 \times 10^{-7} \pm 0,03 \times 10^{-7} \mathrm{a}$ \\
\hline
\end{tabular}

C.V. $=3,40 \%$; DMS = 0,11 e MG = 1,303

MG-Média Geral; CV-Coeficiente de variação e DMS-Desvio mínimo significativo

Obs.: médias seguidas pela mesma letra não diferem estatisticamente pelo teste de Tukey, a $5 \%$ de probabilidade. 
TABELA 5 - Valores de difusividade térmica preditos, experimentais e erros percentuais em relação aos valores experimentais para as polpas integral, $9^{\circ}$ Brix e peneirada.

\begin{tabular}{|c|c|c|c|c|c|c|c|c|c|}
\hline \multirow{3}{*}{ Equações } & \multicolumn{9}{|c|}{ Difusividade Térmica $\left(\times 10^{-7} \mathrm{~m}^{2} / \mathrm{s}\right)$} \\
\hline & \multicolumn{3}{|c|}{ Polpa Integral } & \multicolumn{3}{|c|}{ Polpa $9^{\circ}$ Brix } & \multicolumn{3}{|c|}{ Polpa Peneirada } \\
\hline & $\alpha_{\text {predito }}$ & $\alpha_{\text {exp. }}$ & $\varepsilon_{\mathrm{p}}(\%)$ & $\alpha_{\text {predito }}$ & $\alpha_{\text {exp. }}$ & $\varepsilon_{\mathrm{p}}(\%)$ & $\alpha_{\text {predito }}$ & $\alpha_{\text {exp. }}$ & $\varepsilon_{p}(\%)$ \\
\hline $\begin{array}{l}\text { Alimentos em geral } \\
\text { (RIEDEL, 1969) }\end{array}$ & 1,39 & 1,31 & 6,10 & 1,40 & 1,32 & 6,06 & 1,38 & 1,27 & 8,66 \\
\hline $\begin{array}{l}\text { Alimentos em geral } \\
\text { (MARTENS, citado por } \\
\text { SINGH, 1982) }\end{array}$ & 1,36 & 1,31 & 3,82 & 1,38 & 1,32 & 4,55 & 1,36 & 1,27 & 7,09 \\
\hline $\begin{array}{l}\text { Suco de tomate (CHOI e } \\
\text { OKOS, 1983) }\end{array}$ & 1,30 & 1,31 & 0,76 & 1,34 & 1,32 & 1,52 & 1,29 & 1,27 & 1,57 \\
\hline
\end{tabular}

Na Tabela 6, são mostrados os resultados médios experimentais obtidos para o calor específico das amostras, com variações entre um mínimo de $3,18 \mathrm{~kJ} / \mathrm{kg}^{\circ} \mathrm{C}$ do material peneirado e máximo de $3,71 \mathrm{~kJ} / \mathrm{kg}^{\circ} \mathrm{C}$ das amostras $9^{\circ}$ Brix. Observa-se a correspondência entre os valores de calor específico e o teor de sólidos solúveis. O calor específico das amostras a $9^{\circ}$ Brix difere significativamente do calor específico das amostras integral e peneirada, superando-as em pelo menos 14\%. Já o calor específico das amostras integral e peneirada não diferem significativamente entre si. Com esses resultados infere-se que há uma relação inversa entre o calor específico e o teor de sólidos solúveis, concordando com diversos autores (CHOI e OKOS, 1983; MANOHAR et al., 1991; LAU et al., 1992). Ao mesmo tempo se observa que a redução do teor de sólidos insolúveis do material peneirado não exerceu influência mensurável sobre o calor específico desse material, quando comparado com a polpa integral.

TABELA 6 - Valores médios do calor específico para a polpa de cupuaçu.

\begin{tabular}{|c|c|c|}
\hline Amostra & $\begin{array}{c}\text { Calor Específico } \\
\left(\mathbf{k J} / \mathbf{k g}^{\circ} \mathrm{C}\right)\end{array}$ & Desvio Padrão \\
\hline Polpa integral & $3,24 \mathrm{~b}$ & 0,02 \\
\hline Polpa a $9^{\circ}$ Brix & $3,71 \mathrm{a}$ & 0,04 \\
\hline Polpa peneirada & $3,18 \mathrm{~b}$ & 0,01 \\
\hline \multicolumn{3}{|c|}{$\begin{array}{l}\text { CV = 0,775 \%; DMS = 0,07 e MG = 3,378 } \\
\text { MG-Média Geral; CV-Coeficiente de variação e } \\
\text { DMS-Desvio mínimo significativo } \\
\text { Obs.: médias seguidas pela mesma letra não diferem } \\
\text { estatisticamente pelo teste de Tukey a 5\% }\end{array}$} \\
\hline
\end{tabular}

Na Tabela 7, são mostrados os valores do calor específico das amostras, calculados a partir de equações desenvolvidas por Siebel, citado por Choi e Okos (1986), Dickerson (1968) e Lamb, citado por Choi e Okos (1986). Os calores específicos calculados pelas equações superaram os valores determinados experimentalmente em todas as amostras. Os menores erros percentuais foram verificados para o material $9^{\circ}$ Brix para as três equações, significando que menores proporções de sólidos solúveis viabilizam o uso dessas equações para os cálculos de calor específico, com baixo erro percentual. Os cálculos dos calores específicos dos materiais integral e peneirado utilizando-se as três equações resultaram em valores de erros percentuais que superaram $13 \%$ em todos os casos.

Na Tabela 8 são mostrados os valores de condutividade térmica $(\mathrm{k})$ das polpas, que variaram de 0,42 $\mathrm{W} / \mathrm{m}^{\circ} \mathrm{C}$ a $0,50 \mathrm{~W} / \mathrm{m}^{\circ} \mathrm{C}$, calculados de forma indireta por meio da Equação 5. Esses dados foram obtidos utilizando os valores da massa específica a $30^{\circ} \mathrm{C}$, o valor do calor específico e os valores de difusividade térmica, todos determinados experimentalmente. A partir dos resultados obtidos para a condutividade térmica da polpa do cupuaçu, verifica-se que a redução da concentração dos sólidos solúveis provoca um aumento da condutividade térmica.

Na Tabela 9, são mostrados os valores da condutividade térmica para os três materiais calculados pelas equações de Sweat e Parmelee (1978), Riedel citado por Choi e Okos (1986) e Choi e Okos (1983). Observa-se que os menores erros percentuais ocorreram na amostra a 9oBrix, apresentando valores entre zero e $24 \%$, o que significa que as equações predisse- 
ram o comportamento desse material com precisão entre excelente e razoável. Para as amostras integrais e peneiradas, os menores erros percentuais foram de $11,36 \%$ e $16,67 \%$, respectivamente, poden- do ser considerados razoáveis. Apesar de proposta para margarina e derivados de leite, a equação de Sweat e Parmelee (1978) resultou, em todas as amostras, nos menores erros.

TABELA 7 - Valores de calores específicos preditos, experimentais e erros percentuais em relação aos valores experimentais para as polpas integral, $9^{\circ}$ Brix e peneirada.

\begin{tabular}{|c|c|c|c|c|c|c|c|c|c|}
\hline \multirow{3}{*}{ Equações } & \multicolumn{9}{|c|}{ Calor específico (kJ/kg $\left.{ }^{\circ} \mathrm{C}\right)$} \\
\hline & \multicolumn{3}{|c|}{ Polpa Integral } & \multicolumn{3}{|c|}{ Polpa $9^{\circ}$ Brix } & \multicolumn{3}{|c|}{ Polpa Peneirada } \\
\hline & $\mathrm{C}_{\mathrm{p} \text { pred. }}$ & $C_{p \text { exp. }}$ & $\varepsilon_{\mathrm{p}}(\%)$ & $\mathrm{C}_{\mathrm{p} \text { pred. }}$ & $C_{p \text { exp. }}$ & $\varepsilon_{\mathrm{p}}(\%)$ & $\mathrm{C}_{\mathrm{p} \text { pred. }}$ & $C_{p \text { exp. }}$ & $\varepsilon_{\mathrm{p}}(\%)$ \\
\hline $\begin{array}{l}\text { Produtos alimentícios } \\
\text { (SIEBEL, citado por CHOI } \\
\text { e OKOS, 1986) }\end{array}$ & 3,68 & 3,23 & 13,93 & 3,79 & 3,71 & 2,16 & 3,68 & 3,18 & 15,72 \\
\hline $\begin{array}{l}\text { Produtos alimentícios } \\
\text { (DICKERSON, 1968) }\end{array}$ & 3,80 & 3,23 & 17,65 & 3,89 & 3,71 & 4,85 & 3,80 & 3,18 & 19,50 \\
\hline $\begin{array}{l}\text { Produtos alimentícios } \\
\text { (LAMB, citado por CHOI e } \\
\text { OKOS, 1986) }\end{array}$ & 3,78 & 3,23 & 17,02 & 3,86 & 3,71 & 4,04 & 3,77 & 3,18 & 18,55 \\
\hline
\end{tabular}

TABELA 8 - Valores da condutividade térmica da polpa do cupuaçu.

\begin{tabular}{ccc}
\hline Amostras & Condutividade Térmica $\left(\mathbf{W} / \mathbf{m}^{\mathbf{0}} \mathbf{C}\right)$ & Desvio Padrão \\
\hline Polpa Integral & $0,44 \mathrm{~b}$ & 0,02 \\
Polpa $9^{\circ}$ Brix & $0,50 \mathrm{a}$ & 0,01 \\
Polpa Peneirada & $0,42 \mathrm{~b}$ & 0,01 \\
\hline
\end{tabular}

$\mathrm{CV}=2,88 \%$; MG = 0,452 e DMS = 0,03

MG-Média Geral; CV-Coeficiente de variação e DMS-Desvio mínimo significativo

Obs.: médias seguidas pela mesma letra não diferem estatisticamente pelo teste de Tukey, a $5 \%$ de probabilidade

TABELA 9 - Valores de condutividade térmica determinados pelas equações da literatura e os valores do erro percentual em relação aos dados experimentais.

\begin{tabular}{|c|c|c|c|c|c|c|c|c|c|}
\hline \multirow{3}{*}{ Equações } & \multicolumn{9}{|c|}{ Condutividade térmica $\left(\mathrm{W} / \mathrm{m}^{\circ} \mathrm{C}\right)$} \\
\hline & \multicolumn{3}{|c|}{ Polpa Integral } & \multicolumn{3}{|c|}{ Polpa $9^{\circ}$ Brix } & \multicolumn{3}{|c|}{ Polpa Peneirada } \\
\hline & $\mathbf{k}_{\text {predito }}$ & $\mathbf{k}_{\text {exp. }}$ & $\varepsilon_{\mathrm{p}}(\%)$ & $\mathbf{k}_{\text {predito }}$ & $\mathbf{k}_{\text {exp. }}$ & $\varepsilon_{\mathrm{p}}(\%)$ & $\mathbf{k}_{\text {predito }}$ & $\mathbf{k}_{\text {exp. }}$ & $\varepsilon_{\mathrm{p}}(\%)$ \\
\hline $\begin{array}{l}\text { Sucos de frutas (RIEDEL, } \\
\text { citado por CHOI e OKOS, } \\
\text { 1986) }\end{array}$ & 0,56 & 0,44 & 27,27 & 0,57 & 0,50 & 14,00 & 0,56 & 0,42 & 33,33 \\
\hline $\begin{array}{l}\text { Suco de Tomate (CHOI e } \\
\text { OKOS, 1986) }\end{array}$ & 0,62 & 0,44 & 40,91 & 0,62 & 0,50 & 24,00 & 0,62 & 0,42 & 47,62 \\
\hline $\begin{array}{l}\text { Margarina (SWEAT e } \\
\text { PARMELLEE,1978) }\end{array}$ & 0,49 & 0,44 & 11,36 & 0,50 & 0,50 & 0,00 & 0,49 & 0,42 & 16,67 \\
\hline
\end{tabular}

Ciênc. agrotec., Lavras, v. 28, n. 1, p. 126-134, jan./fev., 2004 


\section{CONCLUSÕES}

As diferenças nos teores de sólidos não influenciaram as difusividades térmicas determinadas nas amostras de polpa de cupuaçu integral, $9^{\circ}$ Brix e peneirada, tendo como valor médio $1,30 \times 10^{-7} \mathrm{~m}^{2} / \mathrm{s}$. As equações de difusividade térmica de Riedel (1969), Martens, citado por Singh (1982) e de Choi e Okos (1983) forneceram bons valores em relação aos dados experimentais em todas as amostras, com erros percentuais variando de $0,76 \%$ a $8,66 \%$.

Os valores obtidos para os calores específicos experimentais das polpas de cupuaçu integral, $9^{\circ} \mathrm{Brix}$ e peneirada foram: $3,24 \mathrm{~kJ} / \mathrm{kg}^{\circ} \mathrm{C}, 3,71 \mathrm{~kJ} / \mathrm{kg}^{\circ} \mathrm{C}$ e 3,18 $\mathrm{kJ} / \mathrm{kg}^{\circ} \mathrm{C}$, respectivamente. Os teores de sólidos solúveis influenciaram em relação inversa os valores dessa propriedade, com as amostras de menores teores $\left(9^{\circ} \mathrm{Brix}\right)$ apresentando maior calor específico. A redução do teor de sólidos insolúveis na amostra peneirada não influenciou o valor dessa propriedade, quando comparado com o obtido para a polpa integral.

Todas as equações utilizadas para calcular o calor específico resultaram em valores superiores aos encontrados experimentalmente e a equação de Siebel, citado por Choi e Okos (1986) resultou nas melhores aproximações em relação aos dados experimentais.

A condutividade térmica experimental das amostras de polpa de cupuaçu integral, $9^{\circ}$ Brix e peneirada apresentou os valores de $0,44 \mathrm{~W} / \mathrm{m}^{\circ} \mathrm{C}, 0,50$ $\mathrm{W} / \mathrm{m}^{\circ} \mathrm{C}$ e $0,42 \mathrm{~W} / \mathrm{m}^{\circ} \mathrm{C}$, respectivamente. A equação de Sweat e Parmelee (1978) resultou na melhor aproximação aos dados experimentais de condutividade térmica para os três materiais.

Os menores erros percentuais dos valores de calor específico e condutividade térmica foram verificados para o material $9^{\circ}$ Brix para as três equações, inferindo-se que menores proporções de sólidos solúveis viabilizam o uso dessas equações com baixos erros.

\section{REFERÊNCIAS BIBLIOGRÁFICAS}

ALVARADO, J. D. Propiedades físicas de frutas. IV: difusividad y conductividad termica efectiva de pulpas. Latin American Applied Research, Ambato, v. 24, n. 1, p. 41-47, 1994.

ARAÚJO, J. L. Determinação das propriedades termofísicas da polpa do cupuaçu (Theobroma grandiflorum Schum.). 2001. 85 f. Dissertação (Mestrado em Engenharia Agrícola) - Universidade Federal da Paraíba, Campina Grande, 2001.
BHOWMIK, S. R.; HAYAKAWA, K. I. A new method for determining the apparent thermal diffusivity of thermally conductive food. Journal of Food Science, Chicago, v. 44, n. 2, p. 469-474, 1979.

CALZAVARA, B. B. G.; MUlleR, C. H.; KAHWAGE, O. N. C. Fruticultura tropical: cupuaçuzeiro cultivo, beneficiamento e utilização do fruto. Belém: EMBRAPA/CPATU, 1984. 101 p.

CHOI, Y.; OKOS, M. R. The thermal properties of tomato juice concentrates. Transactions of the ASAE, Saint Joseph, v. 26, n. 1, p. 305-311, 1983.

CHOI, Y.; OKOS, M. R. Effects of temperature and composition on the thermal properties of foods. In: LEMAUGUER, M.; JELEN, M. Food engineering and process applications: transport phenomena. London: Elsevier Applied Science, 1986. v. 1, p. 93101 .

DICKERSON, R. W. An apparatus for the measurement of thermal diffusivity of foods. Food Technology, Chicago, v. 19, n. 5, p. 198-204, 1965.

DICKERSON, R. W. Thermal properties of foods. In: TRESSLER, D. K.; ARSDEL, W. B. van; COPLEY, M. J. The freezing preservation of foods. 4. ed. Westport: The AVI, 1968. v. 2.

LAU, K. A.; MARCH, A. C.; LO, K. V.; CUMMING, D. B. Physical properties of celery juice. Canadian Agricultural Engineering, Saskatoon, v. 34, n. 1, p. 105-110, 1992.

LEWIS, M. J. Propiedades físicas de los alimentos y de los sistemas de procesado. Zaragoza: Acribia, 1993. 494 p.

MANOHAR, B.; RAMAKRISHNA, P.; UDAYASANKAR, K. Some physical properties of tamarind (Tamarindus indica L) juice concentrates. Journal of Food Engineering, Great Britain, v. 13, n. 4, p. 241-258, 1991.

ÖZISIK, M. N. Basic heat transfer. Tokyo: McGrawHill, 1977. 572 p.

RAO, M. A.; RIZVI, S. S. H. Engineering properties of foods. 2. ed. New York: Marcel Dekker, 1994. $531 \mathrm{p}$. 
RIEDEL, L. Measurements of termal diffusivity of foodstuffs rich in water. Kaltetechnik-Klimatisierung, [S.1.], v. 21, n. 11, p. 315, 1969.

SILVA, F. A. S. C. The ASSISTAT software: statistical assistance. In: INTERNATIONAL CONFERENCE ON COMPUTERS IN AGRICULTURE, 6., 1996, Cancun. Anais... Cancun: American Society of Agricutural Engineers, 1996. p. 294-298.

SIMÕES, R. M. Propriedades termofísicas da polpa de manga. 1997. 73 f. Dissertação (Mestrado em En- genharia de Alimentos) - Universidade Estadual de Campinas, Campinas, 1997.

SINGH, P. Thermal diffusivity in food processing. Food Technology, Chicago, v. 36, n. 2, p. 87-91, 1982.

SWEAT, V. E.; PARMELEE, C. E. Measurement of thermal conductivity of dairy product and margarines. Journal of Food Process Engineering, Trumbull, v. 2, p. $187-197,1978$. 